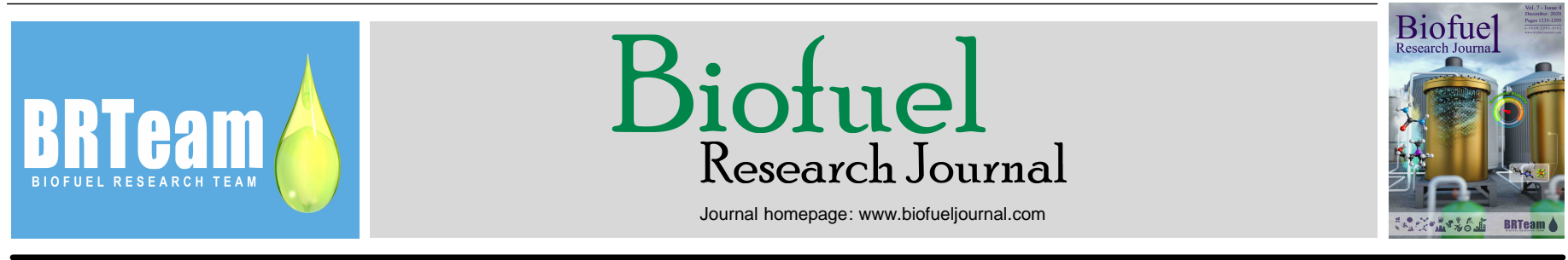

Original Research Paper

\title{
Improving the co-production of triacylglycerol and isoprenoids in Chlamydomonas
}

\author{
Supakorn Potijun ${ }^{1,2}$, Suparat Jaingam ${ }^{1,2}$, Nuttha Sanevas ${ }^{3}$, Srunya Vajrodaya $^{3}$, Anchalee Sirikhachornkit ${ }^{1,2, *}$ \\ ${ }^{I}$ Microalgal Molecular Genetics and Functional genomics Special Research Unit, Department of Genetics, Faculty of Science, Kasetsart University, Bangkok \\ 10900, Thailand. \\ ${ }^{2}$ Center for Advanced Studies in Tropical Natural Resources, National Research University-Kasetsart University (CASTNAR, NRU-KU), Kasetsart University, \\ Bangkok 10900, Thailand. \\ ${ }^{3}$ Department of Botany, Faculty of Science, Kasetsart University, Bangkok 10900, Thailand.
}

\section{HIGHLIGHTS}

$>$ A mutant screening method for improving triacylglycerol (TAG) and isoprenoid content. $>$ Terbinafine-sensitive mutants were isolated in Chlamydomonas.

$>$ One mutant exhibited $2 \mathrm{X}$ higher level of triacylglycerol and $1.5 \mathrm{X}$ higher level of photosynthetic pigments.

$>$ The presence of terbinafine further increased

the triacylglycerol level along with the

accumulation of squalene.

$>$ The mutant exhibited normal growth and photosynthesis.

\section{GRAPHICAL ABSTRACT}

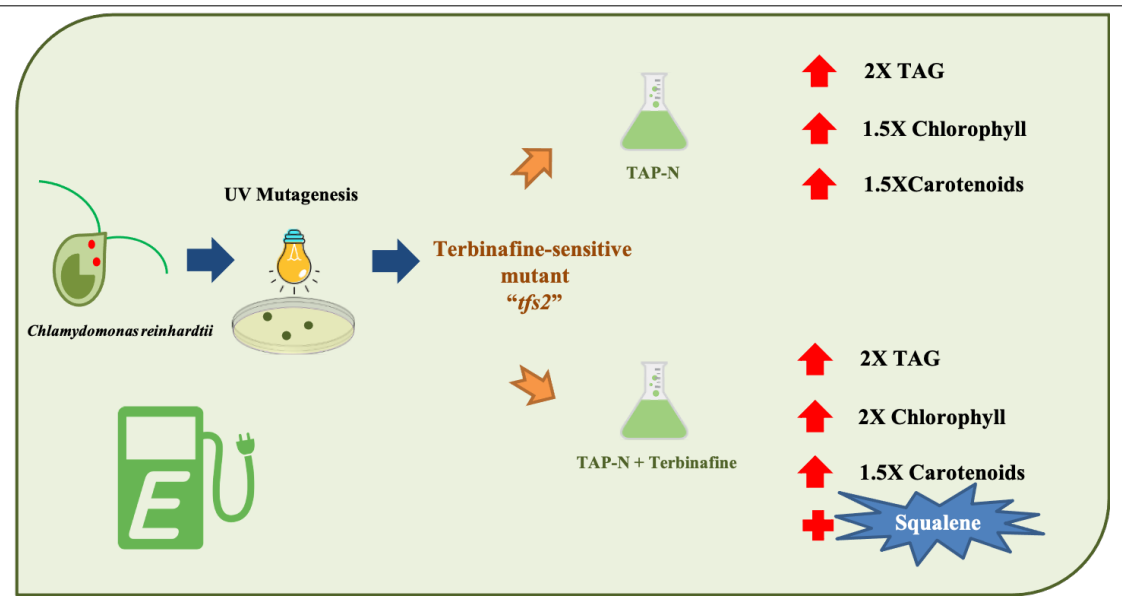

\section{ARTICLE INFO}

\section{Article history:}

Received 1 November 2020

Received in revised form 18 November 2020

Accepted 18 November 2020

Available online 1 December 2020

\section{Keywords:}

Chlamydomonas

Isoprenoid

Microalgae

Squalene

Triacylglycerol

\begin{abstract}
Biodiesel and natural products derived from microalgae require a smaller land area and have higher production rates compared to plants and animals and has recently attracted considerable interest. However, biodiesel production from microalgal triacylglycerol is still far from commercial realization due to its high production cost. One way to overcome this obstacle is to improve the triacylglycerol accumulation and couple its production with other high-value compounds. Of particular interest is the sterol biosynthetic pathway with squalene as an intermediate due to its close relationship with triacylglycerol and carotenoid biosynthetic pathways. Besides, both squalene and carotenoids are isoprenoid lipids that have health benefits. Perturbation of one pathway has been suggested to affect other pathways. Three terbinafine-sensitive mutants of the green microalga Chlamydomonas reinhardtii were isolated using terbinafine, a drug that inhibits squalene epoxidase, leading to squalene accumulation. One of the mutants, $t f s 2$, accumulated twice the amount of wild-type triacylglycerol. As well as squalene accumulation, the presence of terbinafine further increased the triacylglycerol content. The level of prenyl lipid carotenoid and chlorophyll was also more significant than that of the wild type. Growth and photosynthesis were not compromised in this mutant. This is the first study that has demonstrated a mutant screening method to improve the co-production of TAG and isoprenoid lipids in a green microalgae.
\end{abstract}

(c) 2020 BRTeam. All rights reserved.

\footnotetext{
* Corresponding authors at: Tel.: +66 25625444
}

E-mail address: anchalee.si@ku.th

Please cite this article as: Potijun S., Jaingam S., Sanevas N., Vajrodaya S., Sirikhachornkit A. Improving the co-production of triacylglycerol and isoprenoids in Chlamydomonas. Biofuel Research Journal 28 (2020) 1235-1244. DOI: 10.18331/BRJ2020.7.4.2 


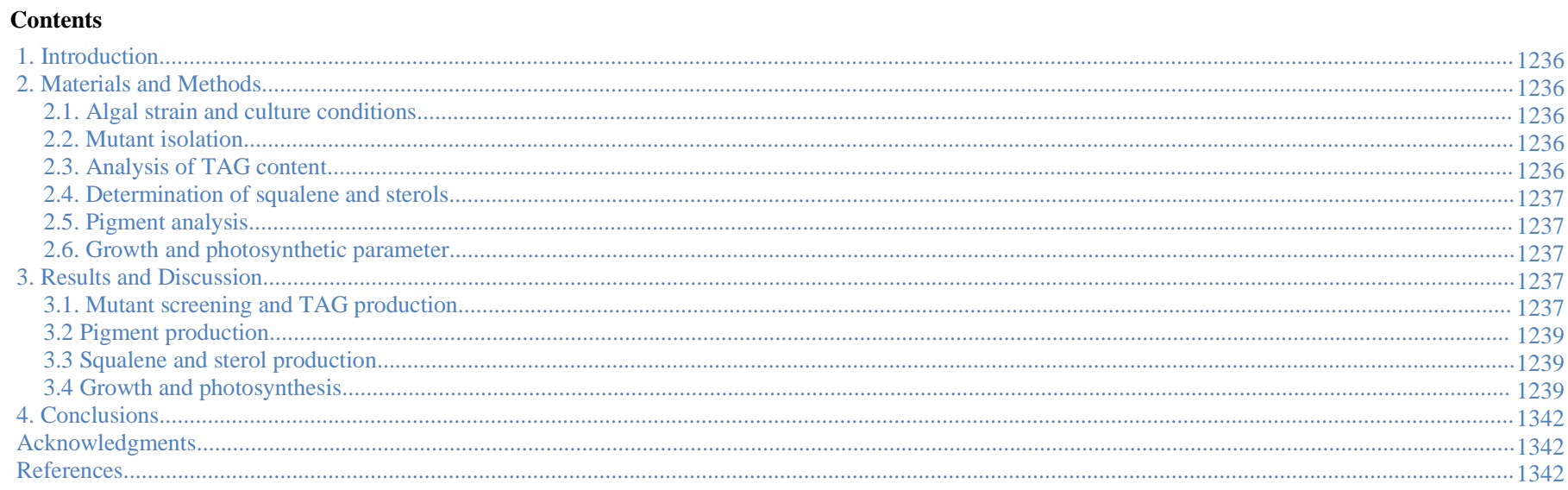

\section{Introduction}

Microalgae have received considerable attention recently as a feedstock with great potential for biofuel production. They have many advantages over land plants, such as higher productivity per area and the ability to grow in wastewater and other unfavorable environmental conditions (Halim et al., 2011; Delrue et al., 2012). However, sustainable industrial production of bioenergy from microalgae has yet to be realized. On the one hand, this is due to limitations at the biological level, such as strain robustness and yield, but on the other hand, it is also caused by technological issues such as the cost of growing, cell harvesting, and lipid extraction.

To bring microalgal biodiesel production a step closer to commercial realization, the production of triacylglycerol (TAG) along with other highvalue products has been suggested (Leu and Boussiba, 2014). Squalene, an intermediate in sterol synthesis, is a polyunsaturated, triterpenic hydrocarbon with nutritional, cosmetic, pharmaceutical, and medical applications. In the cosmetics industry, squalene is present in skincare products due to its ability to hold moisture and its antioxidant activity, which protects cells from free radicals (Huang et al., 2009). It is also used as an ingredient in vaccine adjuvants (Fox, 2009). Currently, the primary source of squalene is shark liver oil. Due to the increasing commercial demand for squalene alongside the growing international concern regarding the exploitation of marine habitats, sustainable sources of squalene are urgently required.

To synthesize TAG, pyruvate is converted to acetyl-CoA, which enters the fatty acid synthetic pathway, generating fatty acids for TAG production (Fig. 1). Through the methylerythritol 4-phosphate (MEP) pathway, pyruvate is used to synthesize isopentenyl diphosphate (IPP), a five-carbon compound that acts as a building block for all isoprenoids. IPP is used to synthesize geralnylgeranyldiphosphate (GGPP), the precursor for photosynthetic pigment chlorophyll and carotenoid, through a series of steps. IPP is also exported from the chloroplasts and enters sterol biosynthesis. Sterols are essential lipids that are important for membrane structure and fluidity. The first committed step of sterol biosynthesis is catalyzed by squalene synthase to synthesize squalene. Squalene is then oxidized by squalene epoxidase. This second enzyme is the target for terbinafine, a drug commonly used in humans and animals to eliminate fungal infection by inhibiting sterol synthesis.

Several studies employing the use of inhibitors for sterol or fatty acid synthesis suggest that a regulatory relationship among these pathways might exist. For instance, in tobacco cells, terbinafine treatment resulted in the accumulation of squalene which was accompanied by an increase in TAG content (Wentzinger et al., 2002). In contrast, the removal of terbinafine restored sterol biosynthesis at the expense of TAG degradation. In the microalga Nannochloropsis oceanica, terbinafine exposure led to an elevated level of free fatty acids (Lu et al., 2014). Treating Chlamydomonas culture with fenpropimorph, a sterol synthesis inhibitor, also induced TAG accumulation (Kim et al., 2015). Furthermore, treating Chlamydomonas with cerulenin, a fatty acid synthase inhibitor, enhanced lutein level (Heredia-Martinez et al., 2018). Similarly, the inhibition of fatty acid synthesis in the microalga Vischeria helvetica resulted in increased carotenoid levels under nitrogendeficient and high-light conditions (Aburai and Abe, 2015).
To explore the possibility of creating a microalgal strain with increased TAG yield along with the production of isoprenoids such as squalene and carotenoid, the green microalga Chlamydomonas reinhardtii was used to screen for terbinafine-sensitive mutants. Three terbinafine-sensitive mutants, i.e., $t f s 1, t f s 2$, and $t f s 3$ were successfully isolated. One of the mutants, $t f s 2$, exhibited improved TAG and pigment production. Under terbinafine treatment, this mutant accumulated squalene at the same level as the wild type (WT). The results suggest that a close relationship exists between TAG, carotenoid, and sterol synthesis and that the manipulation of these pathways can lead to improved product yields.

\section{Materials and Methods}

\subsection{Algal strain and culture conditions}

C. reinhardtii wild-type $4 \mathrm{~A}+$ strain was provided by Prof. Krishna Niyogi (University of California, Berkeley). Cultures were grown in Trisacetate-phosphate $(\mathrm{TAP}+\mathrm{N})$ medium under constant illumination at 35 $\mu \mathrm{mol}$ photons $\mathrm{m}^{-2} \mathrm{~s}^{-1}$ at $25^{\circ} \mathrm{C}$. For the control condition, log-phase cultures were diluted in fresh TAP medium to $2 \times 10^{6}$ cells $\mathrm{mL}^{-1}$ and grown at 50 $\mu \mathrm{mol}$ photons $\mathrm{m}^{-2} \mathrm{~s}^{-1}$ illumination. For nitrogen deprivation conditions, $\log$ phase cultures were washed twice in TAP medium without nitrogen (TAPN) to $2 \times 10^{6}$ cells $\mathrm{mL}^{-1}$. For terbinafine treatment, terbinafine was added to the culture to the final concentration of $10 \mu \mathrm{M}$. For lipids determination, cells were harvested by centrifugation and pellets were kept at $-80^{\circ} \mathrm{C}$ until use.

\subsection{Mutant isolation}

Five milliliters of $C$. reinhardtii at a density of $5 \times 10^{6}$ cells $\mathrm{mL}^{-1}$ were placed in a glass petri dish and kept in darkness for $1 \mathrm{~h}$. The culture was then irradiated under UV light for 30 min using a UV transilluminator (Model M-26, Upland, USA). This treatment resulted in a $0.1-0.2 \%$ survival rate. After overnight incubation in the dark, cells were spread onto TAP medium plates and incubated at 30-35 $\mu$ mol photons $\mathrm{m}^{-2} \mathrm{~s}^{-1}$ illumination at $25^{\circ} \mathrm{C}$. A total of 1,920 colonies were obtained and transferred to fresh TAP medium plates.

For the terbinafine sensitivity test, each strain was patched onto an agar medium containing $0.4-0.8 \mu \mathrm{M}$ of terbinafine, and was incubated for 1-2 weeks. Strains with reduced growth in terbinafine presence were selected for further testing by spotting known numbers of cells onto terbinafinecontaining plates and observing growth compared to the wild type.

\subsection{Analysis of TAG content}

Total lipid was extracted from the cells as described by Bligh and Dyer (1959), using $8 \times 10^{7}$ cells in $4 \mathrm{~mL}$ of chloroform:methanol $(2: 1, \mathrm{v} / \mathrm{v})$. The mixture was shaken manually until the biomass was dispersed in the solvent 


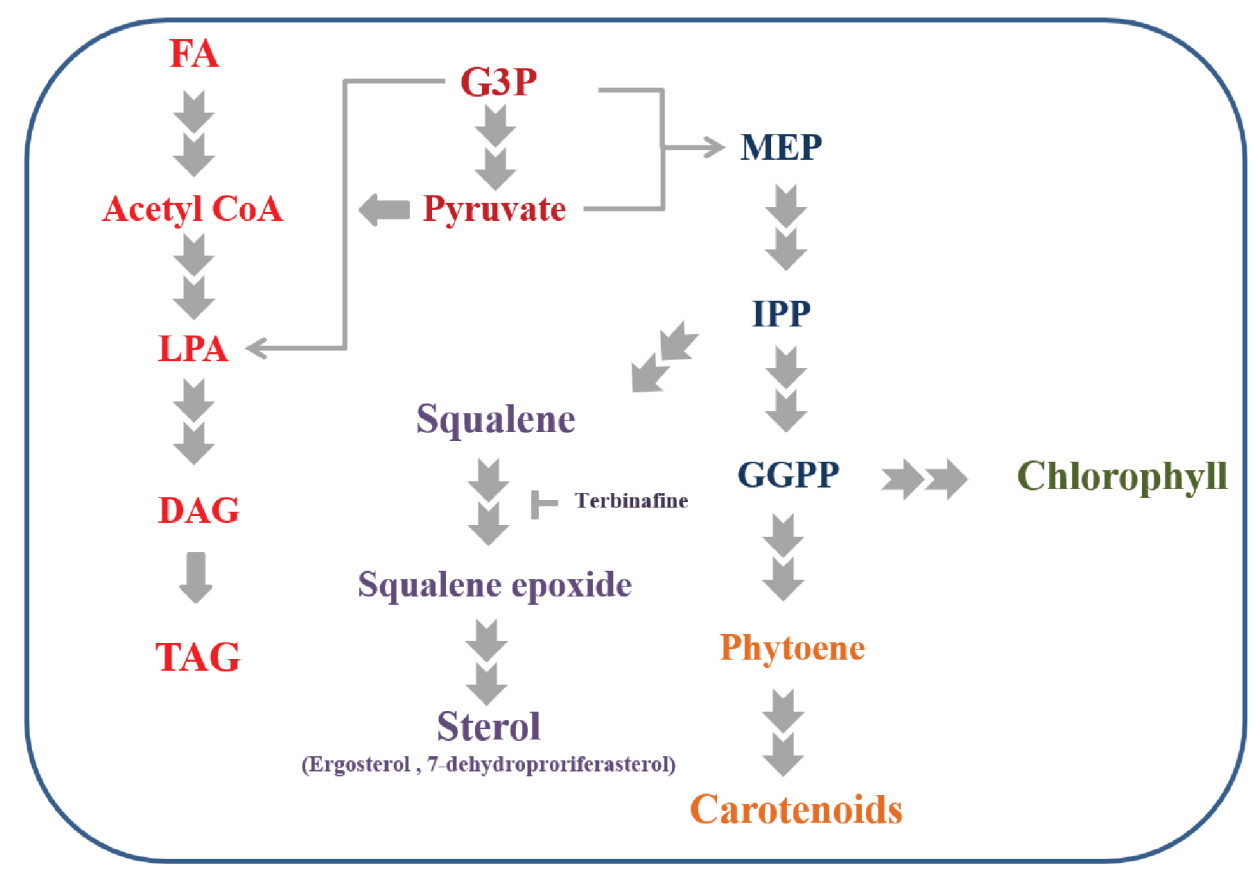

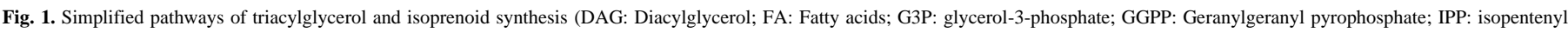
diphosphate; LPA: Lysophosphatidic acid; MEP: 2- $C$-Methyl-d-erythritol 4-phosphate; TAG: Triacylglycerol).

system (Lee et al.,1998). One milliliter of $0.9 \% \mathrm{NaCl}$ was added, and the tube was vortexed. The solution was centrifuged, and the bottom layer was collected using a pasture pipette, dried under nitrogen gas, and kept at $-80^{\circ} \mathrm{C}$ until use.

Two-hundred micrograms of total lipid were spotted onto a $20 \times 20 \mathrm{~cm}$ silica gel 60G F254 TLC plate (Merck, Germany). The plates were developed in hexane:diethyl ether:acetic acid (70:30:1) and stained with iodine vapor. TAG was identified based on the migration of soybean oil (Patel et al., 2014).

Lipid droplet determination was performed by Nile red staining (Kou et al., 2013). Cells were cultured under nitrogen deprivation until a cell density of 1$2 \times 10^{6}$ cells mL ${ }^{-1}$ was reached. Then, $2 \mu \mathrm{L}$ of Nile red and $2.5 \mu \mathrm{L}$ of calcofluor were added to a microfuge tube containing $250 \mu \mathrm{L}$ of cell culture and kept in the dark for $30 \mathrm{~min}$ at room temperature. Two hundred fifty microliters of $8 \%$ formaldehyde were added. Samples were kept in the dark for $30 \mathrm{~min}$ before centrifugation at 12,000 rpm for $10 \mathrm{~min}$. The supernatant was discarded, and the pellet was rinsed twice by $0.5 \mathrm{~mL}$ of $1 \%$ PBS followed by centrifugation. The supernatant was discarded, and $10 \mu \mathrm{L}$ of antifade $(1 \mathrm{~mL}$ of $10 \times$ PBS, $9 \mathrm{~mL}$ of glycerol, and $100 \mu \mathrm{L}$ of $20 \%$ propyl gallate) was then added. Stained cells were visualized under a confocal laser scanning microscope (Nikon Digital Eclipse C2Si, Japan), using the excitation/emission wavelength at $488 / 525 \mathrm{~nm}$. To quantify the relative level of neutral lipids, $950 \mu \mathrm{L}$ of culture at $2 \times 10^{6}$ cells $\mathrm{mL}^{-1}$ was mixed with $50 \mu \mathrm{L}$ of dimethyl sulfoxide and $2.5 \mu \mathrm{L}$ Nile red. Twohundred microliters of each mixture were transferred to a 96-well plate. Fluorescent intensity was measured in arbitrary units by a microplate reader (TECAN SparkControl v1.1.13.0, Switzerland) using the excitation/emission wavelength at $528 / 576 \mathrm{~nm}$.

\subsection{Determination of squalene and sterols}

To quantify the squalene level using high-performance liquid chromatography (HPLC), the total lipid sample was dissolved in $250 \mu \mathrm{L}$ of acetonitrile and filtered through a $0.2 \mu \mathrm{m}$ PTFE filter. The analyses were performed using the Waters HPLC system and a C18 column $(150 \times 3.0 \mathrm{~mm}$, $5 \mu \mathrm{M}$ particle size, Phenomenex, USA). Acetonitrile (100\%) was used as the mobile phase at a flow rate of $1.5 \mathrm{~mL} \mathrm{~min}^{-1}$. Squalene was detected at $195 \mathrm{~nm}$, and was quantified using a previously generated standard curve of peak area $v s$. known squalene amount (Lu et al., 2004).
Sterol content was determined following the method of Kajikawa et al. (2015). The sample was analyzed using a gas chromatography-mass spectrometry (GCMS-QP2020; Shimadzu, Kyoto, Japan) equipped with an DB-5MS capillary column $(30 \mathrm{~m} \times 0.25 \mathrm{~mm}$, film thickness $0.25 \mathrm{~mm}$, Agilent Technologies, Santa Clara, CA), carrier gas: $\mathrm{He}\left(1 \mathrm{~mL} \mathrm{~min}^{-1}\right)$, oven temperature: $150-300^{\circ} \mathrm{C}$ (increase rate $20^{\circ} \mathrm{C} \mathrm{min}^{-1}$ ). The ionization voltage was $70 \mathrm{eV}$, and the scan range was 40-500 Da. The ergosterol and 7 dehydroporiferasterol peaks were identified by their respective standards (Sigma-Aldrich), and the contents were compared by peak areas.

\subsection{Pigment analysis}

The photosynthetic pigments were extracted from $1 \mathrm{~mL}$ of culture using $1 \mathrm{~mL}$ of $80 \%$ acetone. The mixture was vortexed until the pellets were white. The supernatant was assayed spectrophotometrically, and the quantity of the pigment was calculated based on the previously reported formula (Lichtenthaler et al., 1987).

\subsection{Growth and photosynthetic parameter}

Cell growth was monitored by counting cells on a hemacytometer under a microscope. To determine the photosynthesis efficiency expressed by the ratio of $\mathrm{F}_{\mathrm{v}} / \mathrm{F}_{\mathrm{m}}$., one milliliter of the cell culture was incubated in the dark for $15 \mathrm{~min}$ before placing it in a chamber of the Z985 Cuvette AquaPen (Photon systems instrument, Czech Republic).

\section{Results and Discussion}

\subsection{Mutant screening and TAG production}

Following UV mutagenesis, a total of 1,920 colonies were screened on plates containing different concentrations of terbinafine. Three terbinafinesensitive mutants, $t f s 1, t f s 2$, and $t f s 3$ were identified. Their growth was similar to that of the wild type in a normal medium. In contrast, these mutants exhibited extreme sensitivity to $0.8 \mu \mathrm{M}$ of terbinafine (Fig. 2). 

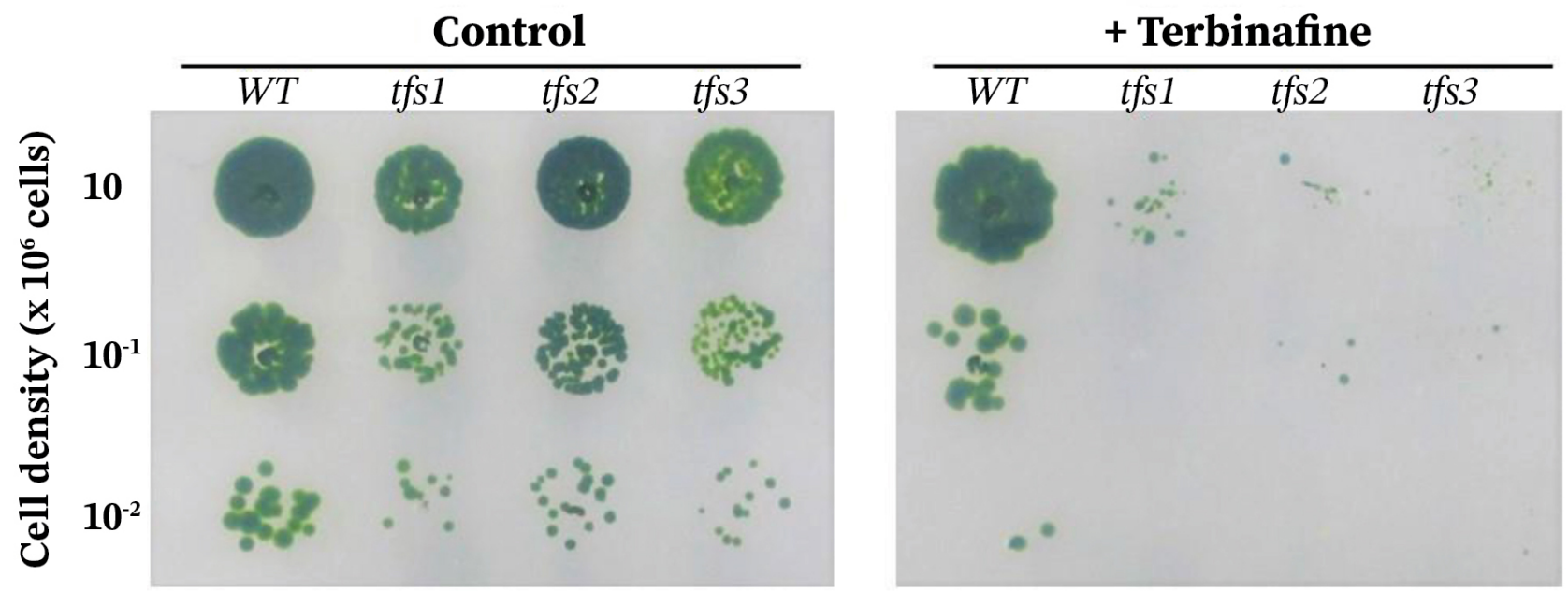

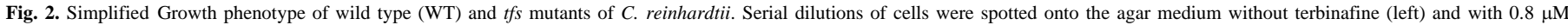
terbinafine (right) and cultivated for 1 week.

Nitrogen starvation is the most widely used condition to induce lipid droplet formation in microalgae. To investigate lipid droplet formation following nitrogen deprivation, the log phase cells of all strains were transferred to a nitrogen-depleted medium. Nile red, a lipophilic fluorescent dye, was used to assay lipid accumulation visually. This dye can detect the neutral lipids in all cellular structures, but the green fluorescence is only observed when the dye penetrates intracellular neutral lipid globules (Cooksey et al., 1987). After two days without nitrogen, $t f s 2$ exhibited the highest fluorescent intensity compared to the other strains (Fig. 3a).

Thin-layer chromatography was then employed to estimate the TAG a
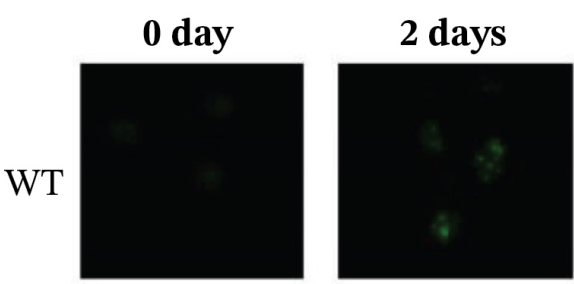

$t f s 1$

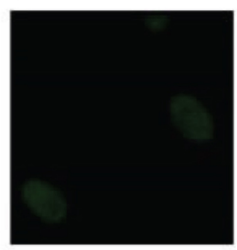

$t f S 2$
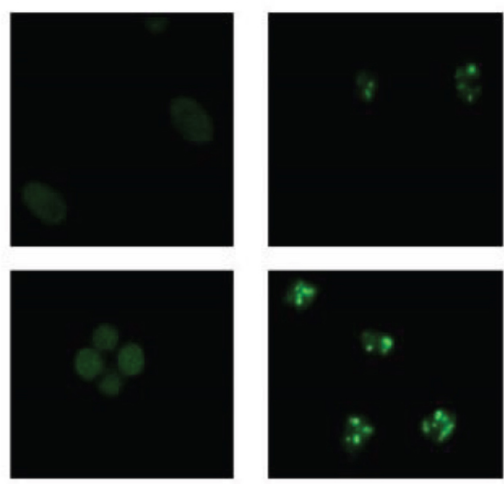

tfs3

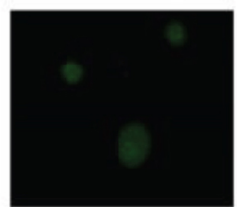

b

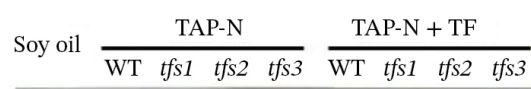

TAG

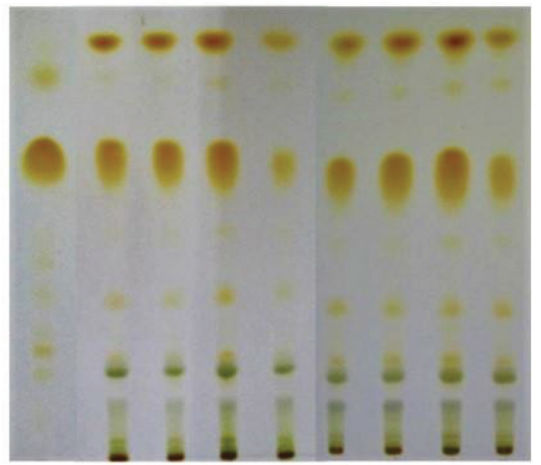

C

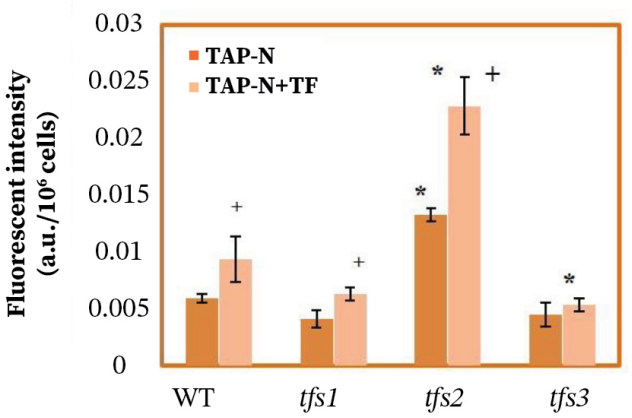

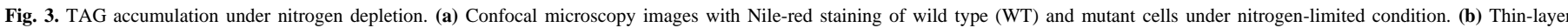

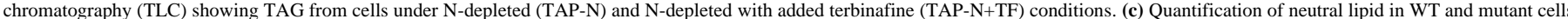

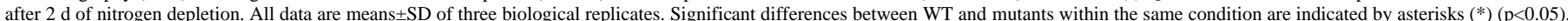
Significant differences between $\mathrm{N}$-depleted $(\mathrm{TAP}-\mathrm{N})$ and $\mathrm{N}$-depleted with added terbinafine (TAP-N+TF) conditions of the same strain are indicated by plus signs $(+)(\mathrm{p}<0.05)$ 
Table 1.

Pigments, squalene, and TAG contents. All data are means \pm SD of three biological replicates. Significant differences between the wild type $(\mathrm{WT})$ and mutant are indicated by asterisks $(*)(\mathrm{p}<0.05)$.

\begin{tabular}{|c|c|c|c|c|c|c|c|c|c|}
\hline & \multirow{2}{*}{ Media } & \multicolumn{2}{|c|}{$\begin{array}{l}\text { Chlorophyll } \\
\left(\mu \mathrm{g} / \mathbf{1 0}^{6} \text { cells }\right)\end{array}$} & \multicolumn{2}{|c|}{$\begin{array}{l}\text { Carotenoids } \\
\left(\mu \mathrm{g} / 10^{6} \text { cells }\right)\end{array}$} & \multicolumn{2}{|c|}{$\begin{array}{c}\text { Squalene } \\
\left(\mathrm{mg} / 10^{6} \text { cells }\right)\end{array}$} & \multicolumn{2}{|c|}{$\begin{array}{c}\text { TAG } \\
\text { (a.u./10 } \text { cells) }\end{array}$} \\
\hline & & WT & $t s \mathrm{~s} 2$ & WT & $t f s 2$ & WT & $t f s 2$ & WT & $t s \mathrm{~s} 2$ \\
\hline \multirow{2}{*}{ TAP } & No TF & $3.53 \pm 0.10$ & $4.86 \pm 0.45^{*}$ & $0.78 \pm 0.08$ & $0.97 \pm 0.15$ & - & - & - & - \\
\hline & $\mathbf{T F}$ & $2.46 \pm 0.12$ & $4.64 \pm 0.61 *$ & $0.52 \pm 0.06$ & $1.05 \pm 0.09 *$ & $0.90 \pm 0.05$ & $0.99 \pm 0.04$ & - & - \\
\hline \multirow{2}{*}{ TAP-N } & No TF & $1.76 \pm 0.10$ & $2.98 \pm 0.70^{*}$ & $0.62 \pm 0.27$ & $1.02 \pm 0.19^{*}$ & - & - & $0.006 \pm 0.0004$ & $0.013 \pm 0.0006 *$ \\
\hline & TF & $1.13 \pm 0.20$ & $2.23 \pm 0.20^{*}$ & $0.59 \pm 0.28$ & $0.83 \pm 0.06 *$ & $0.96 \pm 0.05$ & $1.00 \pm 0.04$ & $0.010 \pm 0.0003$ & $0.023 \pm 0.0046^{*}$ \\
\hline
\end{tabular}

level in these mutants. Besides, to investigate the effect of terbinafine on TAG production, cells were placed in a nitrogen-depleted medium with and without terbinafine for $2 \mathrm{~d}$. Using soy oil as a standard, it was clear that $t f s 2$ showed larger and darker bands than other strains (Fig. 3b). The band from $t f s 2$ in the medium with terbinafine also appeared darker and larger than the band from the cells grown in the medium without terbinafine. Finally, the relative changes in the TAG level were compared based on the intensity of Nile red fluorescence. It is clear that under the 2-d nitrogen deprivation condition, $t f s 2$ accumulated two folds higher TAG level compared to the WT (Fig. 3c and Table 1). Adding terbinafine seemed to produce a slight increase in TAG production in WT and $t f s 1$. Intriguingly, for $t f s 2$, adding terbinafine led to a substantial elevation in the TAG level to around two times that of the WT level in the same condition. These results are in keeping with those of previous studies where several sterol synthesis inhibitors were shown to increase the level of fatty acids or TAG content (Wentzinger et al., 2002; Lu et al., 2014; Kim et al., 2015).

\subsection{Pigment production}

Prenyl lipid carotenoid and chlorophyll are photosynthetic pigments required for the survival of algae. They also possess antioxidant properties. Some carotenoids, such as $\beta$-carotene and astaxanthin, have been commercially produced from microalgae (Panis and Carreon, 2016). Chlorophyll has also been shown to have health benefits (Solymosi and Mysliwa-Kurdziel, 2017). However, under nitrogen-depletion conditions, which induces TAG production, carotenoid and chlorophyll are degraded (Berges et al., 1996; Liefer et al., 2018; Tossavainen et al., 2019), making it difficult to effectively co-produce TAG and carotenoid.

In a normal growth medium, the WT and all the mutants showed similar carotenoid levels (Fig. 4a). Yet when terbinafine was added, the carotenoid level in WT decreased, whereas it stayed the same in both $t f s 2$ and $t f s 3$. Under nitrogen-depletion conditions, the WT, $t f_{s} l$, and $t f_{s} 3$ all had reduced carotenoid levels (Fig. 4b). Only $t f s 2$ exhibited a normal level of carotenoid in this condition, even with the presence of terbinafine (Fig. 4a and Table 1).

The chlorophyll content followed a similar trend to the carotenoid content. In a nitrogen-sufficient condition, all of the mutants showed slightly higher chlorophyll levels than the WT (Fig. 4c). The presence of terbinafine resulted in a reduction in chlorophyll content in the WT. In contrast, terbinafine did not affect chlorophyll content in any of the mutants. Under nitrogen deprivation, the chlorophyll level was significantly reduced in all strains (Fig. 4d). The chlorophyll content in $t f s 2$ was the least affected by this condition, and this mutant was able to maintain the highest level of chlorophyll compared to the other strains.

\subsection{Squalene and sterol production}

Squalene is a key intermediate of sterol biosynthesis. Normally, Chlamydomonas does not accumulate squalene. The genes encoding squalene synthase and squalene epoxidase in Chlamydomonas have been identified and characterized (Kajikawa et al., 2015). Partial knockdown of squalene epoxidase resulted in squalene accumulation without growth inhibition. However, the overexpression of squalene epoxidase in the knockdown strain did not enhance the squalene accumulation.

To explore the possibility of producing squalene along with TAG and to investigate whether or not the mutations affect squalene accumulation, terbinafine was added to the medium with and without nitrogen to determine the squalene level. The WT, $t f s 1$, and $t f s 2$ accumulated similar levels of squalene in both conditions (Fig. 5a and Table 1). Only the tfs 3 mutant showed a lower level of squalene.

The major sterols in Chamydomonas are ergosterol and 7dehydroporiferasterol (Brumfield et al., 2017). To examine whether these mutants are defective in sterol biosynthesis, both major sterols were quantified by GC-MS. The result showed that there were no significant changes in the level of these predominant sterols in the mutants (Fig. 5b).

A total of 109 terbinafine-sensitive strains were identified in a study of terbinafine mutant screening from a deletion mutant collection of $S$. pombe (Fang et al., 2012). The proteins, whose mutations led to terbinafine sensitivity, were from diverse categories. These included; ergosterol biosynthesis, membrane trafficking, histone acetylation and deacetylation, ubiquitination, signal transduction, ribosome biosynthesis and assembly, transcription and translation regulation, cell wall organization and biogenesis, mitochondrion function, amino acid metabolism, nucleic acid metabolism, lipid metabolism, meiosis, and other functions. Therefore, the sensitivity to terbinafine is in fact not limited to a mutation in its target, squalene epoxidase, or in other genes in the sterol pathway, but rather it can be from a mutation in other cellular processes. This phenomenon explains the differences in the phenotypes of Chlamydomonas tfs mutants.

The fact that $t f s 2$ was able to synthesize squalene and sterol normally suggested that its mutation did not affect sterol synthesis per se. However it can be speculated that the mutation in $t f s 2$ could affect some regulatory molecule that involves lipid distribution and composition among the cell membrane, chloroplasts membrane, and lipid droplets. Changes in membrane lipid distribution and composition could lead to terbinafine sensitivity. In fact, it was found that Nannochloropsis oceanica cells treated with terbinafine showed aberrant membrane structure and deformed cells and severely affected chloroplasts (Lu et al., 2014). In Chlamydomonas, treatment with the sterol production inhibitor, fenpropimorph, resulted in the conversion of polar lipid of chloroplast membrane to TAG (Kim et al. 2015). These results demonstrate a close relationship between membrane lipids and storage lipids, which could be used to develop strategies to produce certain types of lipid.

\subsection{Growth and photosynthesis}

Because $t f s 2$ exhibited the most promising phenotype, having increased the TAG and pigment content without affecting the squalene and sterol content, this strain was further examined to determine if the increase in the production of these lipids was compensated for in its growth or photosynthesis. Nitrogen deprivation and the presence of terbinafine were also included in the conditions tested. To summarize, there was no difference in cell growth between the WT and the $t f s 2$ mutant grown in the same condition (Figs. 6a-d). Under the normal conditions, both strains grew rapidly on the first day but showed a slower growth rate between days 
$\mathbf{a}$

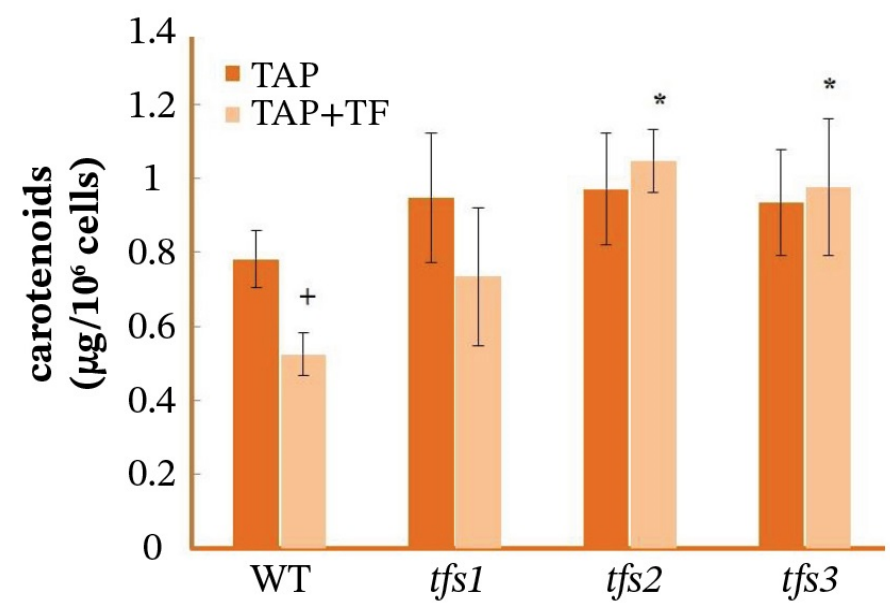

C

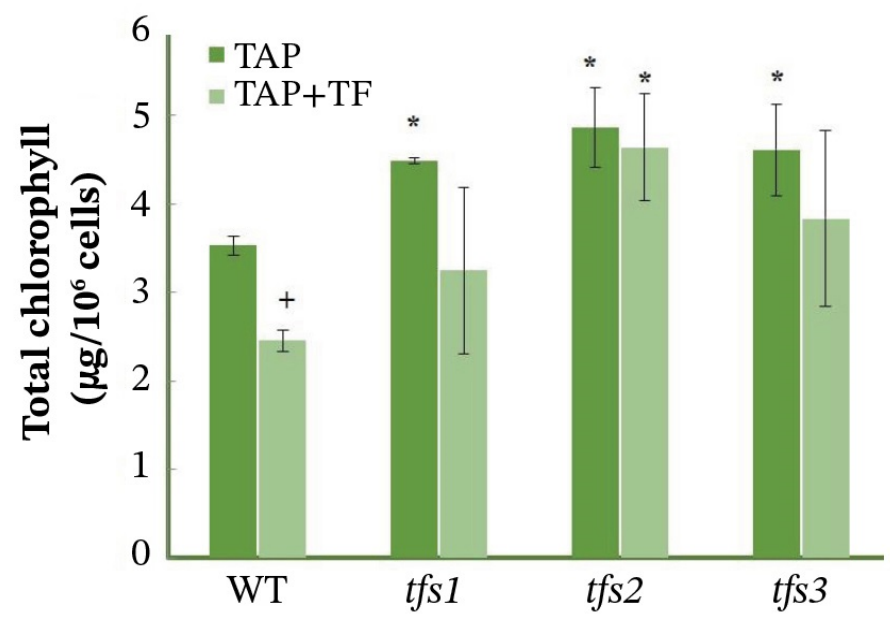

b

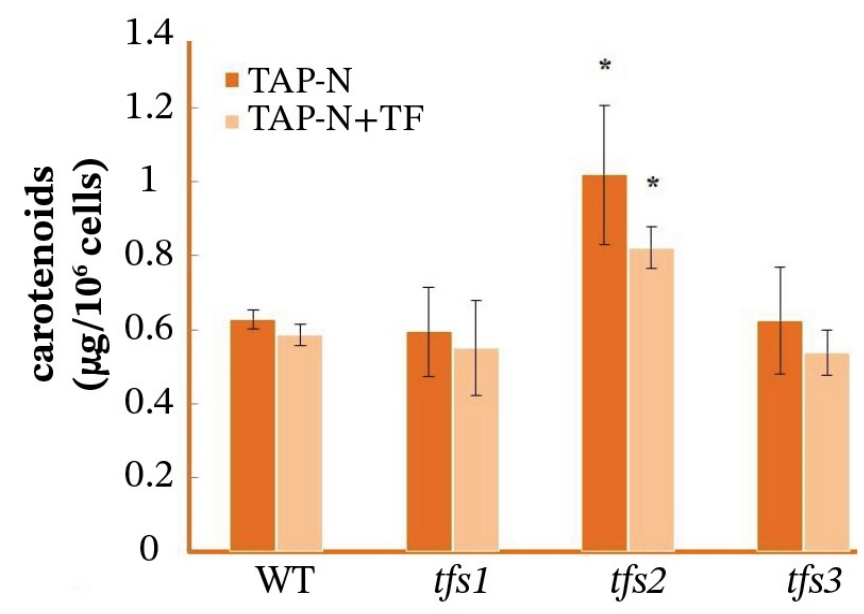

d

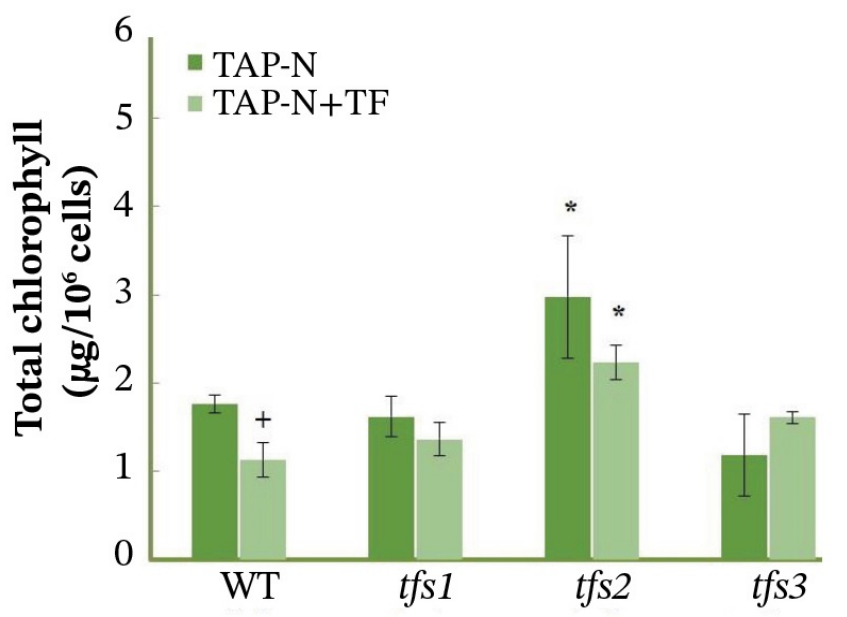

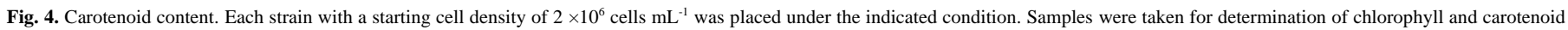

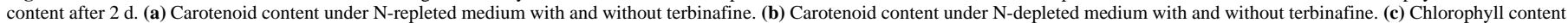

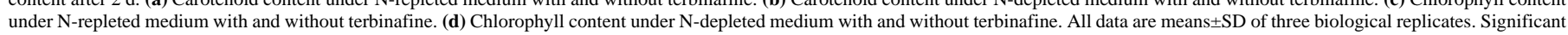

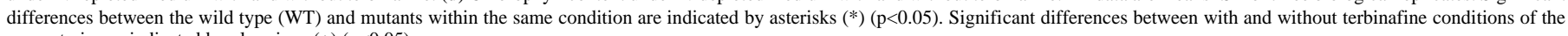
same strain are indicated by plus signs $(+)(\mathrm{p}<0.05)$.

one and four (Fig. 6a). The presence of terbinafine did inhibit growth at the beginning of the experiment, but the cultures were able to reach a similar cell density as the control on day four (Fig. 6b). Nitrogen-limited conditions have been known to inhibit growth because nitrogen is an essential element in the cells. Without inorganic nitrogen supply, protein synthesis will come to a halt. As expected, under the nitrogen-deprivation condition, both the WT and $t f s 2$ grew much slower compared to the control condition (Fig. 6c). Nevertheless, the presence of terbinafine in this condition did not affect cell growth (Fig. 6d).

Light utilization needs to be efficient to maximize algal growth, especially in outdoor conditions where the light intensity changes constantly. The maximum quantum yield $\left(\mathrm{F}_{\mathrm{v}} / \mathrm{F}_{\mathrm{m}}\right)$ of photosystem II (PSII) can be used to monitor the efficiency of light utilization by the photosystem II (PSII) to estimate algal health. In a normal growth medium with and without added terbinafine, $t f s 2$ showed a lower $\mathrm{F}_{\mathrm{v}} / \mathrm{F}_{\mathrm{m}}$ value than the WT at the start of the experiment only (Figs. 7A and B). However, the values recorded for the WT and $t f s 2$ were similar during the rest of the time points. Similarly, under a nitrogen-limited condition, the $\mathrm{F}_{\mathrm{v}} / \mathrm{F}_{\mathrm{m}}$ values of the $t f s 2$ mutant were lower than those of the WT at the beginning of the experiment (Figs. 7C and D). Interestingly the values of $t f s 2$ were significantly higher than those of the
WT from days two to four, in both the medium with and without terbinafine.

Both nitrogen deprivation and treatment with fatty acid or sterol inhibitors can lead to oxidative stress, especially in the chloropla st. This must be contained to prevent cell death. For example, the fatty acid synthesis inhibitor cerulenin caused chloroplast shrinkage and increased reactive oxygen species (ROS) production (Heredia-Martínez et al., 2018). In several studies, terbinafine has also been shown to induce oxidative stress (Francois, 2007; Thevissen et al., 2007; Shekhova et al., 2017). Some evidence suggests that in the green algae, the TAG biosynthesis pathway may play a more active role in the stress response as carbon and energy storage under environmental stress conditions (Hu et al., 2008; Adams et al., 2013; Du and Benning, 2016). Treatment with terbinafine in Saccharomyces led to an increase in lipid droplet size and in the overall TAG level (Garaiová et al., 2014). In the yeast erg1 mutant, a point mutation in $\operatorname{erg} 1$ reduced squalene epoxide activity, leading to squalene accumulation accompanied by a defect in growth (Garaiová et al., 2014). The mutant was hypersensitive to terbinafine. It also accumulated a higher 
$\mathbf{a}$

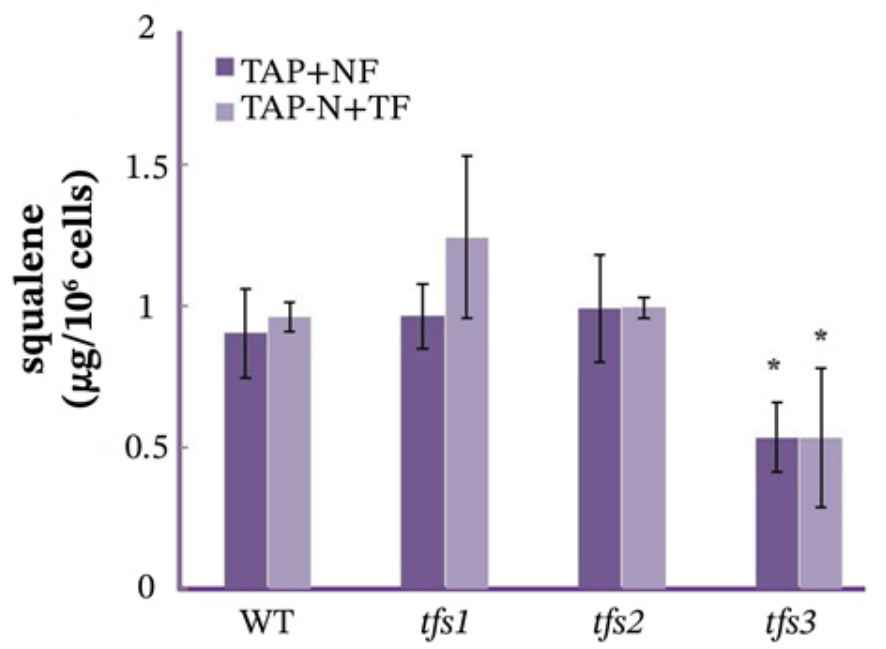

b

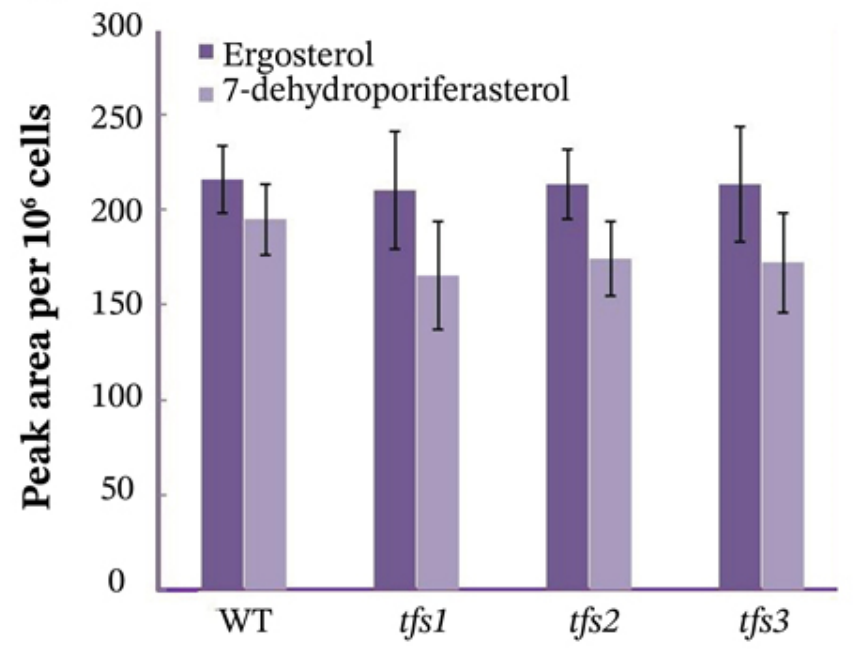

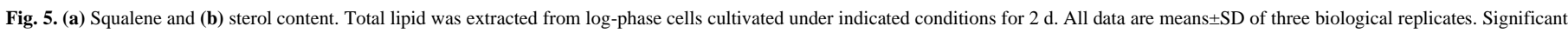
differences between the wild type (WT) and mutants within the same condition are indicated by asterisks $(*)(\mathrm{p}<0.05)$.

\section{$\mathbf{a}$}
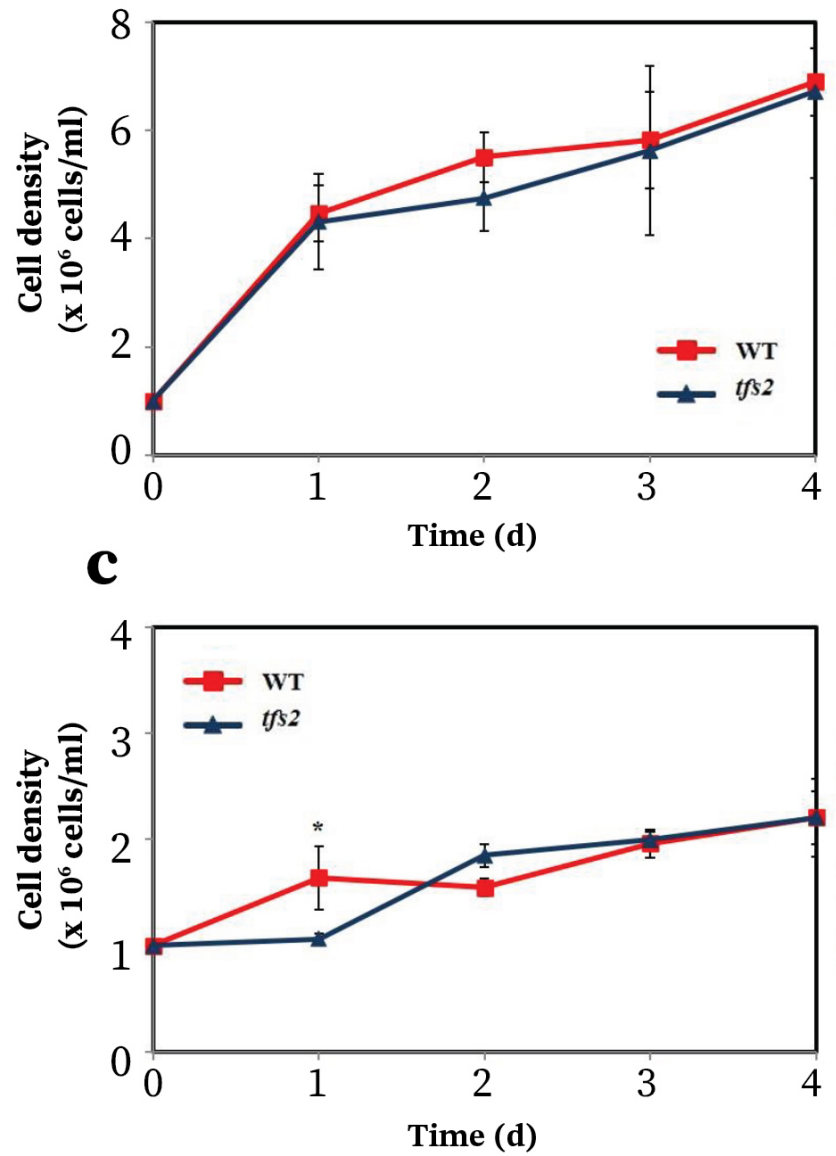

b
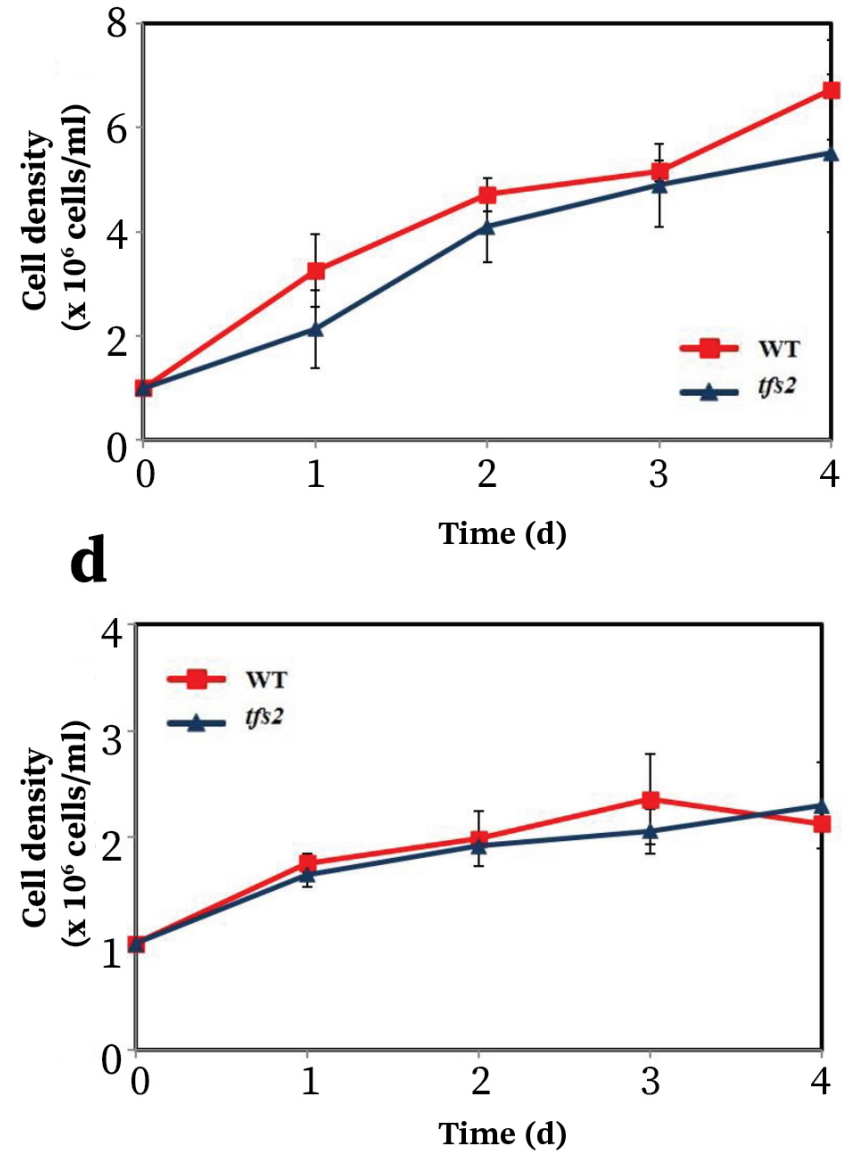

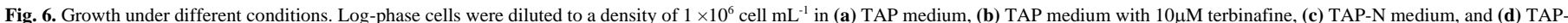

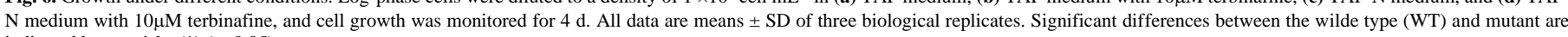
indicated by asterisks $(*)(\mathrm{p}<0.05)$ 

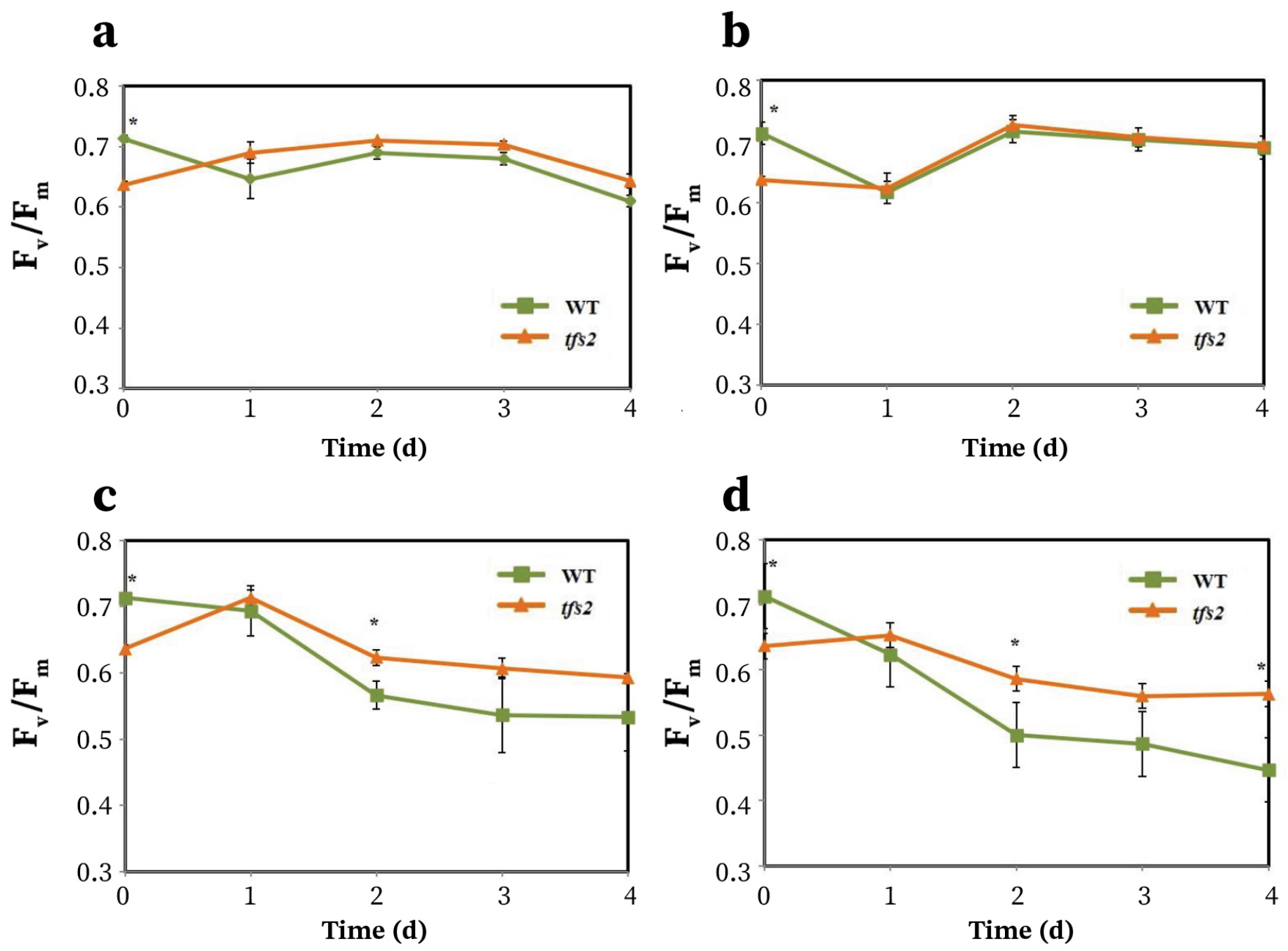

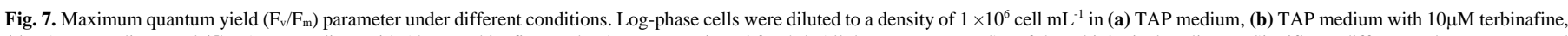

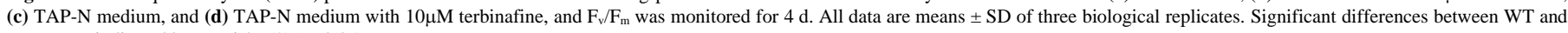
mutant are indicated by asterisks $(*)(\mathrm{p}<0.05)$.

number of lipid droplets with increased size. The fact that the Chlamydomonas tf $r 2$ mutant had increased levels of TAG and carotenoids is beneficial for survival under oxidative stress induced by nitrogen deprivation conditions combined with terbinafine treatment. The significantly higher $\mathrm{F}_{\mathrm{v}} / \mathrm{F}_{\mathrm{m}}$ value of tfs2 under this combination of stress elements implies that its PSII is less damaged by oxidative stress. Therefore, this mutant is more likely to be resilient under stress conditions, which are induced by outdoor cultivation such as high light intensity, high temperature, high salt concentration, etc. Therefore, a similar screening could be performed in other green algae to isolate mutants for the improved production of TAG and isoprenoid in this condition.

\section{Conclusions}

A mutant screening to improve the co-production of TAG and isoprenoids was performed using the green microalga Chlamydomonas reinhardtii, based on a close regulatory relationship between the fatty acid biosynthesis pathway and the isoprenoid synthesis pathway. A terbinafine sensitive mutant, $t f s 2$, was found to accumulate twice as much as the TAG content. The presence of terbinafine, which allowed the cells to accumulate squalene, further increased TAG level by two and a half times compared to the WT. The level of the photosynthetic pigments, chlorophyll, and carotenoid also increased. This mutant exhibited normal growth with higher PSII efficiency under a nitrogen deprivation condition combined with terbinafine treatment compared to the WT. Therefore, it is possible to co-produce TAG and isoprenoids in green algae in this condition. Moreover, such screening could be applied to other species to isolate mutants with increased product yields.

\section{Acknowledgments}

This work was supported by the Graduate Program Scholarship from The Graduate School, Kasetsart University, Kasetsart University Research and Development Institute (KURDI), the Thailand Research Fund (RSA6080030), and by the Faculty of Science, Kasetsart University. We thank Jaruswan Warakanont for her advice.

\section{References}

[1] Aburai, N., Abe, K., 2015. Metabolic switching: synergistic induction of carotenogenesis in the aerial microalga Vischeria helvetica under environmental stress conditions by inhibitors of fatty acid biosynthesis. Biotechnol. Lett. 37(5), 1073-1080.

[2] Adams, C., Godfrey, V., Wahlen, B., Seefeldt, L., Bugbee, B., 2013 Understanding precision nitrogen stress to optimize the growth and 
lipid content trade of in oleaginous green microalgae. Bioresour. Technol. 131, 188-194

[3] Berges, J.A., Charlebois, D.O., Mauzerall, D.C., Falkowski, P.G., 1996. Differential effects of nitrogen limitation on photosynthetic efficiency of photosystems I and II in microalgae. Plant. Physiol. 110, 689-696.

[4] Bligh, E.G., Dyer, W.J., 1959. A rapid method of total lipid extraction and purification. Can. J. Biochem. Physiol. 37, 911-917.

[5] Brumfield, K.M., Laborde, S.M., Moroney, J.V., 2017. A model for the ergosterol biosynthetic pathway in Chlamydomonas reinhardtii. Eur. J. Phycol. 52(1), 64-74.

[6] Cooksey, K., Guckert, J., Williams, S., Callis, P., 1987. Fluorometric determination of the neutral lipid content of microalgal cells using Nile red. J. Microbiol. Meth. 6(6), 333-345

[7] Du, Z.-Y., Benning, C., 2016. Triacylglycerol accumulation in photosynthetic cells in plants and algae, in: Nakamura, Y., Li-Beisson, Y. (Eds.), Lipids in plant and algae development. Springer, Cham, pp. 179205

[8] Delrue, F., Setier, P.A., Sahut, C., Cournac, L., Roubaud, A., Peltier, G., Froment, A.K., 2012. An economic sustainability and energetic model of biodiesel production from microalgae. Bioresour. Technol. 111, 191-200

[9] Fang, Y., Hu, L., Zhou, X., Jaiseng, W., Zhang, B., Takami, T., Kuno, T., 2012. A genomewide screen in Schizosaccharomyces pombe for genes affecting the sensitivity of antifungal drugs that target ergosterol biosynthesis. Antimicrob. Agents Chemother. 56(4), 1949-1959.

[10] Fox, C., 2009. Squalene emulsions for parenteral vaccine and drug delivery. Molecules. 14, 3286-3312

[11] Francois, I., 2007. Investigating the role of reactive oxygen species in the mechanism of action of miconazole and effects on the treatment of diaper dermatitis. J. Am. Acad. Dermatol. 56, AB77.

[12] Garaiová, M., Zambojová, V., Šimová, Z., Griač, P., Hapala, I., 2014. Squalene epoxidase as a target for manipulation of squalene levels in the yeast Saccharomyces cerevisiae. FEMS Yeast Res. 14(2), 310-323.

[13] Halim, R., Gladma, B., Danquah, M.K., Webley, P.A., 2011. Oil extraction from microalgae for biodiesel production. Bioresour. Technol. $102,178-185$

[14] Heredia-Martínez, L.G., Andrés-Garrido, A., Martínez-Force, E., PérezPérez, M.E., Crespo, J.L., 2018. Chloroplast damage induced by the inhibition of fatty acid synthesis triggers autophagy in chlamydomonas. Plant. Physiol. 178, 1112-1129.

[15] Hu, Q., Sommerfeld, M., Jarvis, E., Ghirardi, M., Posewitz, M., Seibert, M., Darzins, A., 2008. Microalgal triacylglycerols as feedstocks for biofuel production: perspectives and advances. Plant. J. 54, 621-639.

[16] Huang, Z.R, Lin, Y.K, Fang, J.Y, 2009. Biological and pharmacological activities of squalene and related compounds: potential uses in cosmetic dermatology. Molecules. 14(1), 540-554

[17] Kajikawa, M., Kinohira, S., Ando, A., Shimoyama, M., Kato, M., Fukuzawa, H., 2015. Accumulation of squalene in a microalga Chlamydomonas reinhardtii by genetic modification of squalene synthase and squalene epoxidase genes. PLoS One. 10(3), e0120446.

[18] Kim, H., Jang, S., Kim, S., Yamaoka, Y., Hong, D., Song, W.Y., Nishida, I., Li-Beisson, Y., Lee, Y., 2015. The small molecule fenpropimorph rapidlyconverts chloroplast membrane lipids to triacylglycerols in Chlamydomonas reinhardtii. Front. Microbiol. 6, 54.

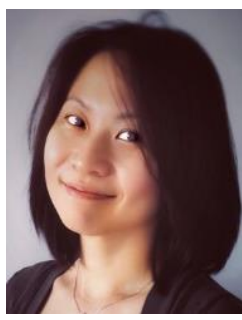

Dr. Anchalee Sirikhachornkit is an Assistant Professor at Kasetsart University (Thailand). She received her Ph.D. in Microbiology from the University of California at Berkeley (USA). Her research interests include microalgal biotechnology and microalgal stress response.
[19] Kou, Z., Bei, S., Sun, J., Pan, J., 2013. Fluorescent measurement of lipid content in the model organism Chlamydomonas reinhardtti. J Appl. Phycol. (25), 1633-1641.

[20] Lee, S.J., Yoon, B.D., Oh, H.M., 1998. Rapid method for the determination of lipid from the green algae Botryococcus braunii. Biotechnol. Tech. 553, 556.

[21] Leu, S., Boussiba, S., 2014. Advances in the production of high-value products by microalgae. Ind. Biotechnol. 10, 169-183.

[22] Lichtenthaler, H.K., 1987. Chlorophylls and carotenoids: Pigments of photosynthetic biomembranes. Methods. Enzymol. 148, 350-382

[23] Liefer, J.D., Garg, A., Campbell, D.A., Irwin, A.J., Finkel, Z.V. 2018. Nitrogen starvation induces distinct photosynthetic responses and recovery dynamics in diatoms and prasinophytes. PLoS One. 13 e0195705.

[24] Lu, H.T., Jiang, Y., Chen, F., 2004. Determination of squalene using high-performance liquid chromatography with diode array detection. Chromatographia. 59, 367-71

[25] Lu, Y., Zhou, W., Wei, L., Li, J., Jia, J., Li, F., Smith, S.M., Xu, J., 2014. Regulation of the cholesterol biosynthetic pathway and its integration with fatty acid biosynthesis in the oleaginous microalga Nannochloropsis oceanica. Biotechnol. Biofuels. 7(1), 81 .

[26] Panis, G., Carreon, J.R., 2016. Commercial astaxanthin production derived by green alga Haematococcus pluvialis: a microalgae process model and a techno-economic assessment all through production line. Algal. Res. 18, 175-190.

[27] Patel, A., Pravez, M., Deeba, F., Pruthi, V., Singh, R.P., Pruthi, P.A., 2014. Boosting accumulation of neutral lipids in Rhodosporidium kratochvilovae HIMPA1 grown on hemp (Cannabis sativa Linn) seed aqueous extract as feedstock for biodiesel production. Bioresour. Technol. 165, 214-222.

[28] Shekhova, E., Kniemeyer, O., Brakhage, A.A., 2017. Induction of mitochondrial reactive oxygen species production by itraconazole terbinafine, and amphotericin B as a mode of action against Aspergillus fumigatus. Antimicrob. Agents Chemother. 61 (11), e00978-17.

[29] Solymosi, K., Mysliwa-Kurdziel, B., 2017. Chlorophylls and their derivatives used in food industry and medicine. Mini. Rev. Med. Chem. 17, 1194-222.

[30] Thevissen, K., Ayscough, K.R., Aerts, A.M., Du, W., De Brucker, K. Meert, E.M.K., Ausma, J., Borgers, M., Cammue, B.P.A., François, I.E.J.A., 2007. Miconazole induces changes in actin cytoskeleton prior to reactive oxygen species induction in yeast. J. Biol. Chem. 282 (30), 21592-21597.

[31] Tossavainen, M., Ilyass, U., Ollilainen, V., Valkonen, K., Ojala, A., Romantschuk, M., 2019. Influence of long termnitrogen limitation on lipid, protein and pigment production of Euglena gracilisin photoheterotrophic cultures. Peer J. 7, e6624

[32] Wentzinger, L.F., Bach, T.J., Hartmann, M.A., 2002. Inhibition of squalene synthase and squalene epoxidase in tobacco cells triggers an up-regulation of 3-hydroxy-3-methylglutaryl coenzyme A reductase. Plant. Physiol. 130(1), 334-346.

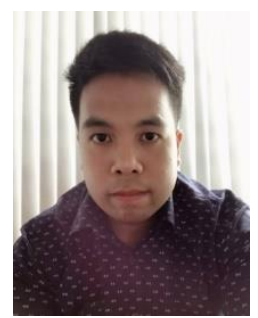

Supakorn Potijun holds a bachelor's degree in Biotechnology from the King Mongkut's Institute of Technology Ladkrabang (Thailand). $\mathrm{He}$ is currently pursuing his master's degree in Genetics at Kasetsart University. His research interests include algal molecular genetics, biochemistry, and biotechnology. 


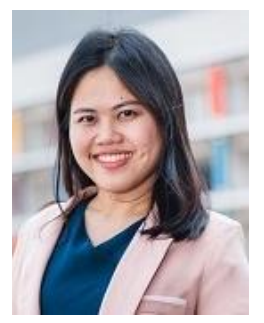

Suparat Jaingam graduated with a bachelor's degree in Biotechnology from the King Mongkut's Institute of Technology Ladkrabang (Thailand) and a master's degree in Genetics from Kasetsart University (Thailand). She is currently working at the Faculty of Medicine Siriraj Hospital, Mahidol University (Thailand) as a research associate of Siriraj Fetal Therapy Center (SiFTC).

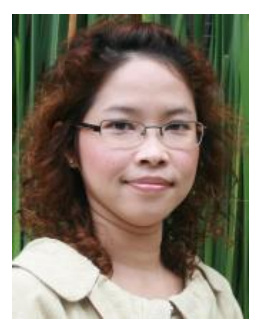

Dr. Nuttha Sanevas is an Assistant Professor at Kasetsart University (Thailand). She received her Ph.D. in Biotechnology from the University of Tsukuba (Japan). Her research area is the diversity, physiology, and utilization of cyanobacteria and freshwater microalgae.

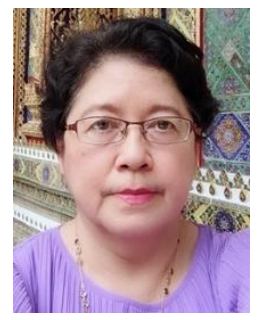

Dr. Srunya Vajrodaya is an Associate Professor at Kasetsart University (Thailand). She received her Dr. rer. nat. in Botanik from the University of Vienna (Austria). Her research interests include plant taxonomy and phytochemistry. 